\title{
Optimization of cultural conditions for Labyrinthula species isolated from mangrove leaves
}

\author{
Jade Angelou S. Dee ${ }^{1,2}$, Angelica Anne V. Ferrer ${ }^{1,2}$, Julie Ann S. Pastor ${ }^{1,2}$, Marine Christine P. Gacelo ${ }^{1,2,3}$ \\ and Gina $R$. Dedeles ${ }^{* 1,2,3}$
}

\begin{abstract}
Labyrinthulids which are marine osmoheterotrophic, straminipilan protists under Labyrinthulomycetes, thrive in brackish waters and mangrove-rich areas. Despite their long history and widespread occurrence in marine and terrestrial environments, this group of organisms is less studied compared to their closely-related organisms such as thraustochytrids, and so far less is known about their optimum growth conditions in laboratory setups. Although the labyrinthulid group is important ecologically, our understanding of their true culturable diversity, isolation, and long-term viability remains poorly documented. Thus, three labyrinthulid strains (AK1, DV1, and DV2) were studied here for culture optimization, mode of growth and prolonged cell viability (at least $14 \mathrm{~d}$ ) by using customized agar media. Optimal temperature $\left(35^{\circ} \mathrm{C}\right)$ and $\mathrm{pH}$ conditions $(\mathrm{pH}$ 6.0-7.0) were best for their growth specifically on media with $6 \%$ glucose concentration and $60 \%$ salinity (parts per thousand). Broth medium with carabao grass extract was suitable in keeping the isolates viable for more than $14 \mathrm{~d}$ but their growth in extract-based media was not significantly different from those grown on base medium, Glucose-Yeast Extract-Peptone agar (GPYA). Although it is not well understood what defense mechanism this organism needs to provide them longer viability in the environment, this study gives insights on their preferred culturable conditions.
\end{abstract}

Keywords: culture medium, Labyrinthulids, microbiology, mycology, Protista

\section{Introduction}

The genus Labyrinthula is one of the three distinct protistan groups of the Labyrinthulomycetes along with aplanochytrids and thraustochytrids. Labyrinthulomycetes belongs to the recently established supergroup Chromalveolata (Leaño \& Damare 2012) under the Phylum Heterokonta and Class Labyrinthista as defined by Raghukumar (2002). Labyrinthulids are marine microorganisms which are prevalent on decaying leaves of mangroves (Perveen et al. 2006, Yokochi et al. 2001). These microorganisms are predominantly saprotrophic, although a few have been reported to be parasitic

${ }^{1}$ Department of Biological Sciences, College of Science, University of Santo Tomas, España, Manila 1008, Philippines

${ }^{2}$ Laboratory of Pure and Applied Microbiology, Research Center for the Natural and Applied Sciences, Thomas Aquinas Research Complex, University of Santo Tomas, España, Manila 1008, Philippines

${ }^{3}$ The Graduate School, University of Santo Tomas, España, Manila 1008

Philippines

*Corresponding email: grdedeles@ust.edu.ph

Date Submitted: 12 August 2019

Date Accepted: 04 April 2020 on a number of invertebrates such as mollusks (Raghukumar 2002). They were originally classified as fungi based on their biflagellate zoospores but were later shown to be distinct making them unrelated to either fungi or Protozoa (Sparrow 1973, Chamberlain \& Moss 1988).

Labyrinthulomycetes is known for its ectoplasmic nets, which are extensions of the plasma membrane associated with the organelle bothrosome or sagenogenetosome (Raghukumar 2002), and zoospore formation (Leaño \& Damare 2012). The groups under this class can be morphologically distinguished from each other (Leander et al. 2004) and/or by molecular analysis. For example, labyrinthulids are characterized as having spindle-shaped cells that are enrobed by ectoplasmic nets (EN) while thraustochytrids are characterized by having unilateral non -motile forms that are not connected by EN. Aplanochytrids, on the other hand, are characterized by having cells which glide through their ectoplasmic nets that do not completely enrobe the cells (Leander et al. 2004). All three groups of these microorganisms are osmoheterotrophic in their mode of nutrition (Leander \& Porter 2001).

Published studies on labyrinthulids in general are scarce and favor monoxenic culture methods. As stated by Wahid et al. 
(2007), the difficulty of maintaining an axenic culture impedes the further exploration of the organism. Current methods of culturing labyrinthulids prove that these species are fastidious in the laboratory since the isolates must be transferred to a fresh medium every 10-14 d. Conventional practices involve subculturing the organism every 2-4 d using a base medium, Glucose-Peptone-Yeast-Extract-Agar (GPYA). However, these organisms tend to slowly lose their viability with continuous subculturing on fresh media.

Recently published methods require a monoxenic culture, serum-based media and/or other media components which are difficult to acquire to ensure their growth longevity. Further, all of these components are costly (Sakata et al. 2000, Yokochi et al. 2001, Kumon et al. 2005, Yadagiri et al. 2012). Therefore, this study aimed to isolate local labyrinthulids and to determine their optimum growth conditions, as well as how to sustain their growth in a cost-effective agar media that could be used for long-term storage of more than $14 \mathrm{~d}$.

\section{Materials and Methods}

\section{Sampling and Isolation}

Sampling sites were chosen on the basis of abundance of mangrove trees in the area and the water source being brackish in nature. The chosen sampling sites were Kalibo, Aklan $\left(11.6892^{\circ} \mathrm{N}, 122.3674^{\circ} \mathrm{E}\right)$ and Davao del Sur bordering the Gulf of Davao $\left(6.6440^{\circ} \mathrm{N}, 125.3473^{\circ} \mathrm{E}\right)$.

Labyrinthula sp. occurs in greater frequency towards the later stages of leaf decomposition (Raghukumar 2002), therefore in this study the fallen senescent mangrove leaves submerged in brackish waters were collected. Samples were placed into Ziplock ${ }^{\circledR}$ plastic bags, separated according to collection sites and transported in an icebox to the laboratory for processing. The $\mathrm{pH}$ and temperature of the sampling sites were noted for the optimization of the culture conditions for the growth of the organism. The leaf samples were cut into strips of about $1 \mathrm{~cm}$ in width and washed twice with sterilized $50 \%$ salt concentration of artificial seawater (50\% ASW), following the procedure of Kumon et al. (2005). Labyrinthulomycetes is exclusively marine and prefers salinities of 20-34 ppt (parts per thousand). They have an absolute requirement for sodium ions which cannot be replaced by potassium ions. Thus, $50 \%$ salt concentration of ASW was used for their growth medium.

The cut and washed leaf samples were aseptically placed on Glucose-Peptone-Yeast Extract-Agar (GYPA) for isolation. GPYA was prepared with glucose $(10 \mathrm{~g} / \mathrm{L})$, peptone $(1.5 \mathrm{~g} / \mathrm{L})$, yeast extract $(1.5 \mathrm{~g} / \mathrm{L})$, agar $(15 \mathrm{~g} / \mathrm{L}), 50 \%$ salt concentration ASW (approximately $1.5 \% \mathrm{NaCl}$ ) to yield a total medium salinity of $50 \%$.
The $\mathrm{pH}$ and temperature used in the optimized culture media were those noted at the sampling sites of the collected leaves to mimic the original environment where the organism was isolated. The plates were sealed with Parafilm ${ }^{\circledR}$ to prevent contamination and incubated until there were positive growth of labyrinthulids.

\section{Purification of Isolates}

Colonies on the media showing typical characteristics of Labyrinthula sp. (i.e., EN formation, spindle-shaped cells under the microscope) (Yokochi et al. 2001, Raghukumar 2002) were picked and subcultured on fresh agar media. After confirmation of their morphology, sub-culturing by agar block followed by taking some cells from the edge of the labyrinthulid colony until pure isolates were obtained. The anti-fungal Nystatin (Orostat, Pharma-Surrey International Incorporated, Quezon City, Philippines) was used in some plates with persistent yeast contaminants (1 drop/100 mL of agar media), in order to obtain pure colonies.

\section{Growth Optimization}

Following purification and identitifcation, the isolates were further inoculated on GPYA media using the agar block method. Growth responses were tested against four environmental parameters, namely $\mathrm{pH}$, incubation temperature, glucose concentration, and salinity. There were four levels of each parameter tested in triplicates: temperature $\left(25,30,35\right.$, and $40^{\circ}$ C); salinity $(40,50,60$, and $70 \% \mathrm{v} / \mathrm{v})$; initial $\mathrm{pH}$ of the agar medium (5.0, 6.0, 7.0, and 8.0), and glucose concentration (3, 5, 7 , and $9 \%$ ). Each parameter was tested separately with the other parameters kept constant (temperature $30^{\circ} \mathrm{C} ; 50 \%$ salinity at $\mathrm{pH}$ 6.0). Results were analyzed using one-way analysis of variance (ANOVA) at $0.5 \%$ significance level using the Data Analysis in Microsoft Excel Office Software 2007 (Microsoft Corporation, Redmond, WA).

\section{Growth in Extract-based Media}

The media used for optimization were GPYA, MGPYA (GPYA with mangrove leaves extract), and CGPYA (GPYA with carabao grass extract). The extracts were prepared by boiling $20 \mathrm{~g}$ of mangrove leaves or carabao grass (Paspalum sp.) in $1.0 \mathrm{~L}$ distilled water for $15 \mathrm{~min}(2.0 \% \mathrm{w} / \mathrm{v})$. Apportioned $50 \%$ volume of the media contained the boiled extracts while the $50 \%$ was ASW. Salinity (3.5\%) was adjusted by adding marine salt $(35 \mathrm{~g} / \mathrm{L})$. The diameter of growth on each plate was measured in the agar using a Vernier caliper. Results were analyzed using one-way analysis of variance (ANOVA) at $0.5 \%$ significance level using the Data Analysis in Microsoft Excel Office Software 2007 (Microsoft Corporation, Redmond, WA). 


\section{Viability Testing}

For qualitative testing of viability in leaf extracts, pure isolates were inoculated in carabao grass extract media (CE) where $0.15-0.20 \mathrm{~g}$ of 2-cm long pieces of carabao grass were placed in vials of $10 \mathrm{~mL} \mathrm{50 \%} \mathrm{ASW} \mathrm{and} \mathrm{then} \mathrm{autoclaved.} \mathrm{The}$ carabao grass pieces were then aseptically inoculated onto GPYA to check for their growth which must exceed the 14-day mark.

\section{Results and Discussion}

\section{Isolation and Purification of Labyrinthulids}

The growth of the labyrinthulid isolates was observed under the microscope showing the spindle-shaped cell body which was lined and plunged into the GYPA medium. However, the ectoplasmic net (EN) of this organism was visually notable by bringing up the agar plate against a light source and observing areas that have a root-like system made up of colorless aggregated cells. Aside from this, the whole EN resembled a dried patch of water that left a mark on the agar medium. The use of this visual method aided in determining their growth using the base isolation plates and all throughout the entire phase of purification. The Labyrinthula spp. found in the samples obtained from Kalibo, Aklan and Davao del Sur are shown in Figure 1 and their growth conditions are shown in Table 1.

Table 1. Labyrinthulid isolates cultured from fallen senescent mangrove leaves from Aklan and Davao samples

\begin{tabular}{cccc}
\hline $\begin{array}{c}\text { Isolate } \\
\text { code }\end{array}$ & Sampling Site & Temperature $\left({ }^{\circ} \mathbf{C}\right)$ & $\mathbf{p H}$ \\
\hline AK1 & Kalibo, Aklan & $34{ }^{\circ} \mathrm{C}$ & $6.0-6.5$ \\
DV1 & Davao del Sur Gulf & $33^{\circ} \mathrm{C}$ & 6.7 \\
DV2 & Davao del Sur Gulf & $33^{\circ} \mathrm{C}$ & 6.7 \\
\hline
\end{tabular}

Several methods such as agar block, point inoculation, and multiple interrupted streaking as shown in Table 2 were used to isolate and purify the organism efficiently. Of these methods, agar block was the most effective for transferring labyrinthulid cells onto a fresh agar medium. Owing to their specific spindle shape and presence of EN, the labyrinthulids are easily recognizable. Thus, the important aspect of using the agar block method is its versatility since it is easy to alter and cut its size to avoid the visibly unwanted yeast colonies. Because of the presence of labyrinthulid cells on the agar block, the cells continued to multiply on fresh agar medium. The desired growth of the organism was observed to be more stable using this agar block technique than the other methods tested.

Table 2. Methods for the subculturing and purification of labyrinthulid isolates

\begin{tabular}{cc}
\hline Methods & Growth \\
\hline Agar Block & + \\
Submarine Agar Block & - \\
Point Inoculation & - \\
Multiple Interrupted Streaking & - \\
Half-strength GPYA Culture & slow growth \\
Full-strength GPYA with anti-fungal & slow growth \\
Seawater Agar Culture & slow growth \\
\hline
\end{tabular}

In addition, submarine agar block (small square piece of inoculum with $5 \mathrm{~mm}$ length and width, on which labyrinthulids had already grown is pushed inward on a V-shaped agar cut of the fresh medium) also induced the growth and multiplication of the target organism. This method somehow also aided in inhibiting the proliferation of the other non-target organisms, but proved to be difficult at the extraction, purification, and growth stages of the labyrinthulid cells.

For the isolation and purification steps, streaking with the use of an inoculating loop proved to be better than using an inoculating needle by point inoculation, to pick up and purify labyrinthulid cells. The latter method produced the least desirable results with no growth of the target organism. This was particularly observed on original plates with mixed growth of yeast and labyrinthulid cells. The needle could pick up the yeast cells growing on top of the labyrinthulid cells making it difficult to take the target cells underneath. Using this point inoculation technique, no labyrinthulid cells and visible EN could be seen under the microscope.

Meanwhile, the multiple interrupted streaking technique was shown to be a good alternative in separating and isolating labyrinthulid cells from the yeast contaminants, even with minimal to heavy contamination. After a few days of incubation, EN were observed forming around the line of streaks thereby differentiating the growth of yeast and labyrinthulid cells on the medium. Here, using an agar block technique helped purify the labyrinthulid cells but still with minimal yeast contaminants.

Plates with positive growth of labyrinthulids were further subcultured onto GPYA. However, separating the yeast from the 


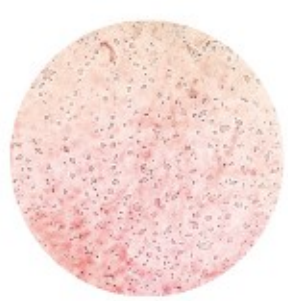

AK1 (100X)
DV1 (400X)

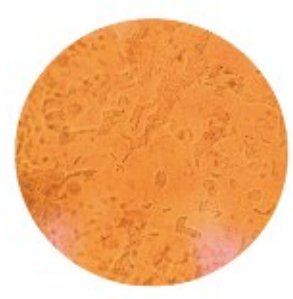

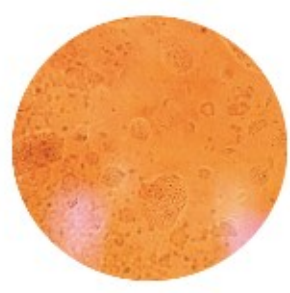

DV2 (400X)

Figure 1. Microscopic and morphological characteristics of Labyrinthula spp. The colony morphology varies in cell size and cell shape from semi-rounded to spindle shape and the ability of cells to penetrate the agar surface. The isolates form into clusters forming circular colonies while others behave in a linear fashion.

labyrinthulid cells was difficult, since the two organisms have grown close to each other. Therefore, different agar types were used alongside GPYA to further the purification process.

Among the three types of agar media tested, the fullstrength GPYA with anti-fungal Nystatin was able to inhibit and slow down the growth of the yeast cells giving more time for the labyrinthulid cells to spread their EN. However, the addition of anti-fungal compound has also affected the growth of labyrinthulids. On the other hand, using half-strength GYPA did not speed up the growth of labyrinthulids nor minimize the presence of contaminants.

The results varied when sea water agar was used with a salinity of $50-100 \%$. Growth rates in general were slow (more or less $2 \mathrm{~d}$ ), the isolates barely grew producing few cells, while pseudohyphal yeast surrounded the perimeter of the agar block and prevented the labyrinthulids to spread their EN. In other plates, growth of the target organism was observed when they were transferred to a fresh half-strength GPYA medium, but the cells were no longer capable of cell multiplication.

In other instances, continuous subculturing with the use of agar block method proved to be the best way to purify the organisms but their viability is lost over successive transfers. To overcome this problem, inclusion of media supplement (i.e. carabao grass leaves) to mimic their natural environment was then attempted.

\section{Growth Optimization}

Labyrinthula sp. has been documented to show versatility in various environments even at extreme conditions. Their ubiquitous presence contributes to their notoriety as plant parasites and high adaptive capability (Raghukumar 2002). However, most studies concerning their optimization conditions in a laboratory setting were done in other countries with temperate climate (Kumon et al. 2002). Since the Philippines is a tropical country, it is expected that the optimal temperature for this organism would be higher than those reported in other studies (Leander \& Porter 2001, Bigelow et al. 2005, Martin et al. 2009, Garcia-Bonet et al. 2011, Bockelmann et al. 2012, Lindholm et al. 2016, Bennett et al. 2017, Wang et al. 2019).

As to growth (Fig. 2), all of the isolates proliferated during the first $24 \mathrm{~h}$, noting a rapid increase in cell density within a relatively short period of time. During the $30^{\text {th }}$ hour (more than $1 \mathrm{~d})$ until around the $72^{\text {nd }}$ hour $(4 \mathrm{~d})$, cell growth started to fluctuate showing little to no growth at all. This behavior was observed on three isolates except for DAV2 where growth started to decline as early as the $60^{\text {th }}$ hour $(3 \mathrm{~d})$ until it has reached the death phase.

In addition, all isolates proliferated best at $35^{\circ} \mathrm{C}$ (Fig. 3) which was close to the conditions where they were sampled
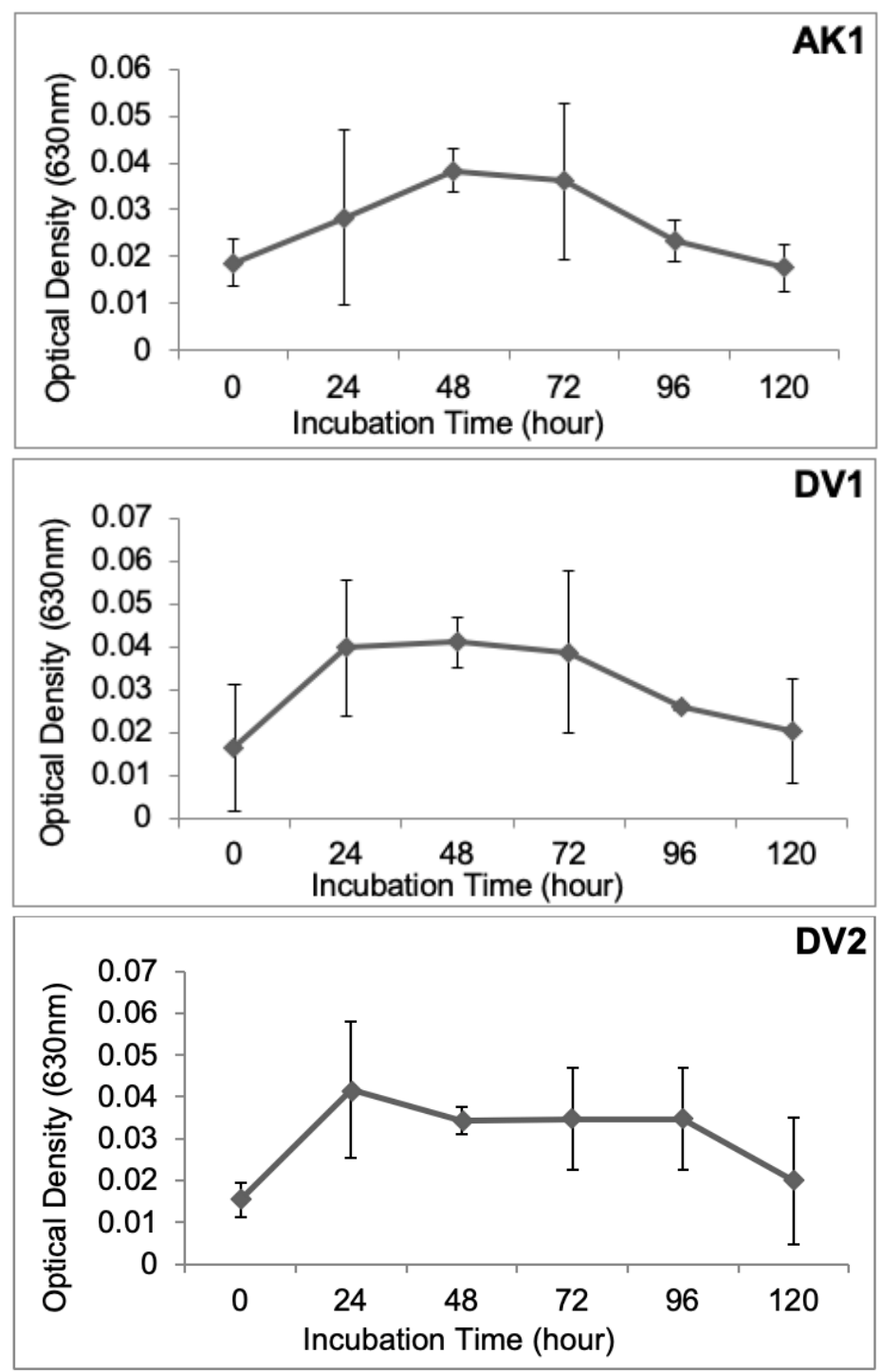

Figure 2. Growth curve of 3 isolates (AK1, DV1 and DV2) on basal medium, GPY for $120 \mathrm{~h}$. Error bars indicate standard deviation $(\mathrm{n}=3)$ $(\mathrm{p}>0.05)$. 


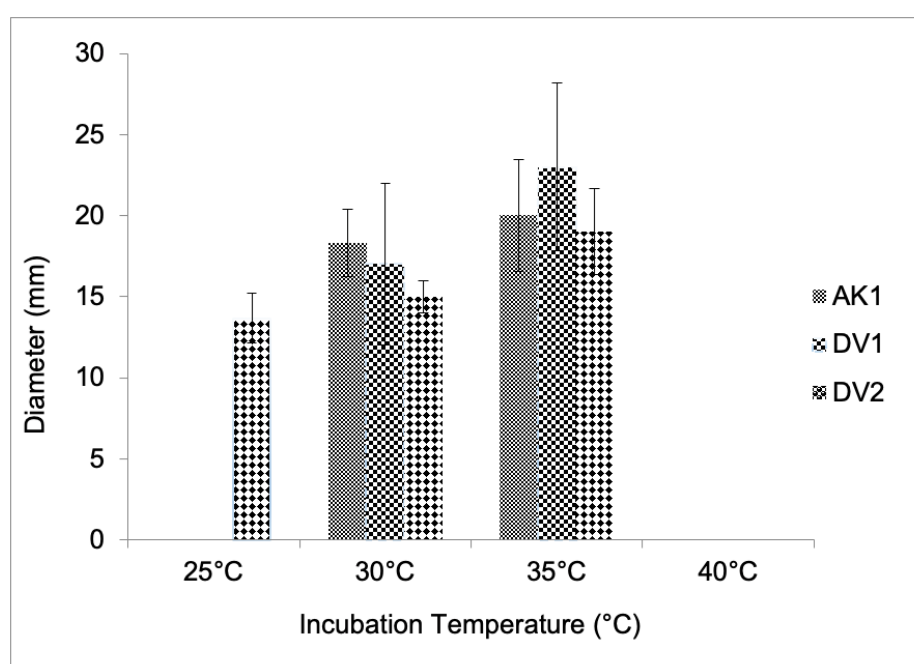

Figure 3. Temperature optimization on basal medium, GPYA. Error bars indicate standard deviation $(n=3)(p>0.05)$.

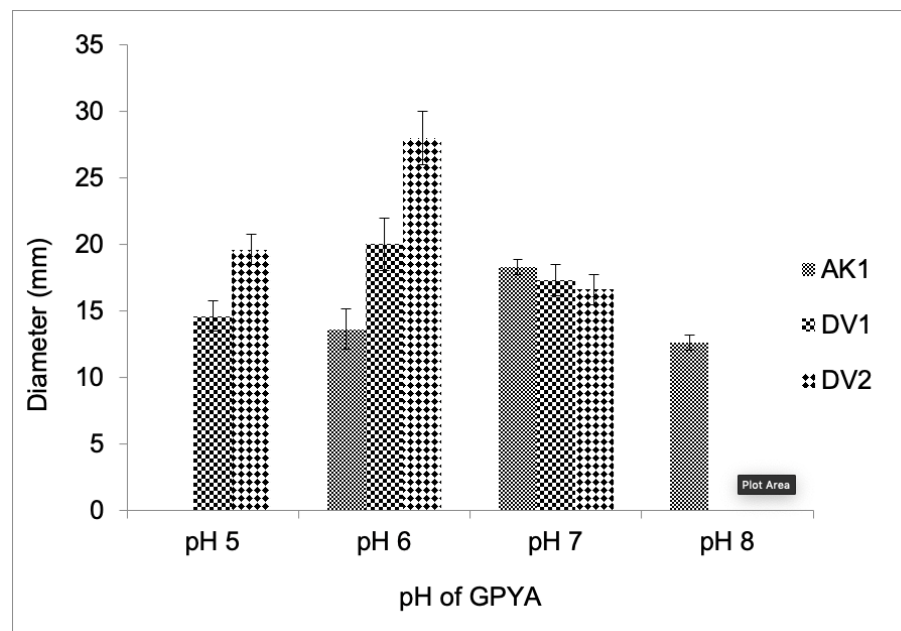

Figure 4. $\mathrm{pH}$ optimization on basal medium, GPYA. Error bars indicate standard deviation $(\mathrm{n}=3)(\mathrm{p}>0.05)$. The optimum $\mathrm{pH}$ for growth ranges from 6.0 to 7.0 showing their adaptability to $\mathrm{pH}$ fluctuations. Growth were also noted at an acidic $\mathrm{pH}$ (5.0) and basic $\mathrm{pH}(8.0)$.

(around $33-35^{\circ} \mathrm{C}$ ). Growth was still observed at room temperature, although slower particularly for isolate DV2. At $40^{\circ} \mathrm{C}$, no growth was observed.

The preferred $\mathrm{pH}$ (mean $\mathrm{pH}=6.0$ ) of the organism was determined (Fig. 4). Overall, isolate AK1 was the most versatile to $\mathrm{pH}$ fluctuations, showing growth at $\mathrm{pH} 5.0$ to 8.0. As observed, labyrinthulid isolates seemed more adaptive to $\mathrm{pH}$ fluctuations rather than on varying temperature ranges. This optimal $\mathrm{pH}$ was also similar to the recorded $\mathrm{pH}$ of their sampling sites between $\mathrm{pH}$ 6.0-7.0.
Optimum glucose concentration was assessed to determine the proper amount of glucose supplement necessary for their growth. Data on Fig. 5 shows that Labyrinthula isolates could best proliferate on culture media with $6 \%(\mathrm{w} / \mathrm{v})$ glucose concentration and even at concentrations lower than this. Two isolates (DV1 and DV2) grew best at 7\% glucose concentration while AK1 at 5\%. On the other hand, the optimal salinity (50$60 \%$ ASW) (Fig. 6) favored all isolates. Only isolate DV2 did not grow at $70 \%$ ASW. The Labyrinthula isolates showed versatility in environments with high salinity, since all had exhibited growth at $100 \%$ on Sea Water Agar during the purification stage.

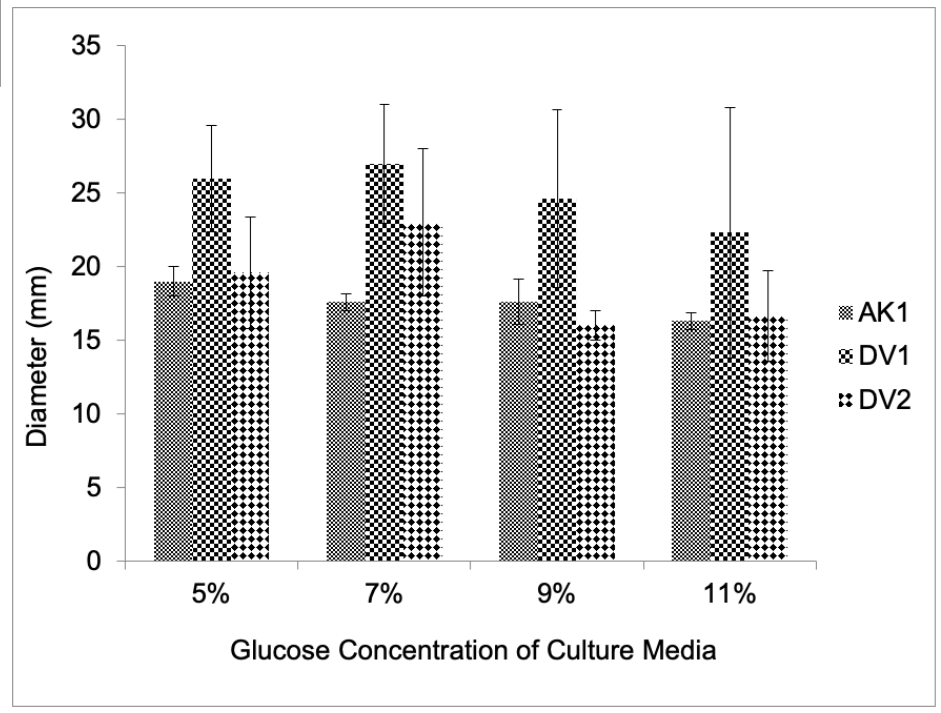

Figure 5. Glucose optimization on basal medium, GPYA. Error bars indicate standard deviation $(\mathrm{n}=3)(\mathrm{p}>0.05)$.

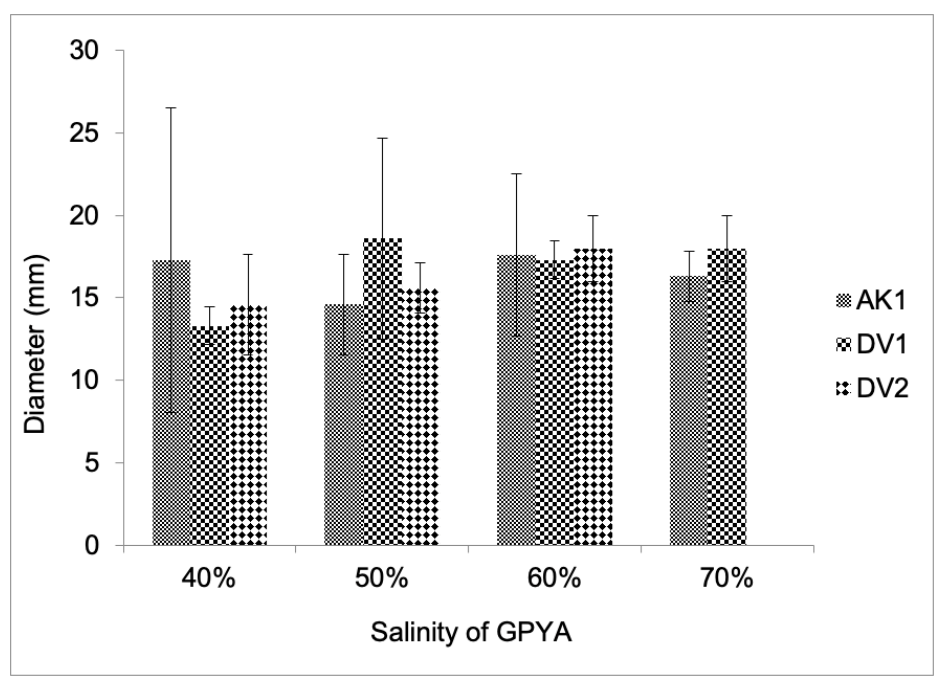

Figure 6. Salinity optimization on basal medium, GPYA. Error bars indicate standard deviation $(\mathrm{n}=3)(\mathrm{p}>0.05)$. 
Table 3. Second viability test for Labyrinthula (AK1, DV1, and DV2) cells

\begin{tabular}{ccccc}
\hline Isolate & Status & Date inoculated into vial & Date subcultured & $\begin{array}{c}\text { Confirmation of } \\
\text { growth at } 35^{\circ} \mathrm{C}\end{array}$ \\
\hline AK1 & Pure & $10 / 24$ & $2 / 8$ & $2 / 9$ \\
DV1 & Pure & $10 / 24$ & $2 / 8$ & $2 / 9$ \\
DV2 & Pure & $11 / 28$ & $2 / 8$ & $2 / 9$ \\
\hline
\end{tabular}

\section{Viability Testing}

To ensure the viability of both pure and under purification Labyrinthula isolates (Tables 2 and 3), sterile vials containing $10 \mathrm{~mL}$ of distilled water with $50 \%$ seawater were prepared with the addition of pieces (blades) of carabao grass. Prior to inoculation of labyrinthulid cells on the broth medium, leaf pieces were washed twice with sterilized $50 \%$ ASW. The cut samples were then placed onto the broth medium and sterilized. Labyrinthula species were inoculated on these vials and after 24 months of incubation, the isolates were subcultured on fresh media and surprisingly these were able to grow and multiply. Revival of the organisms using this method was easy. Growth was observed after 24 and $96 \mathrm{~h}$ of incubation at room temperature and after $24 \mathrm{~h}$ using the optimal temperature $\left(35^{\circ} \mathrm{C}\right)$. The long lag phase was observed on isolates from Davao (DV1 and DV2) and better growth was further observed in the second trial.

Another interesting observation on the growth of isolates after revival was the formation of their EN visible to the naked eye. The EN formations were seen on the sides of the glass vial.
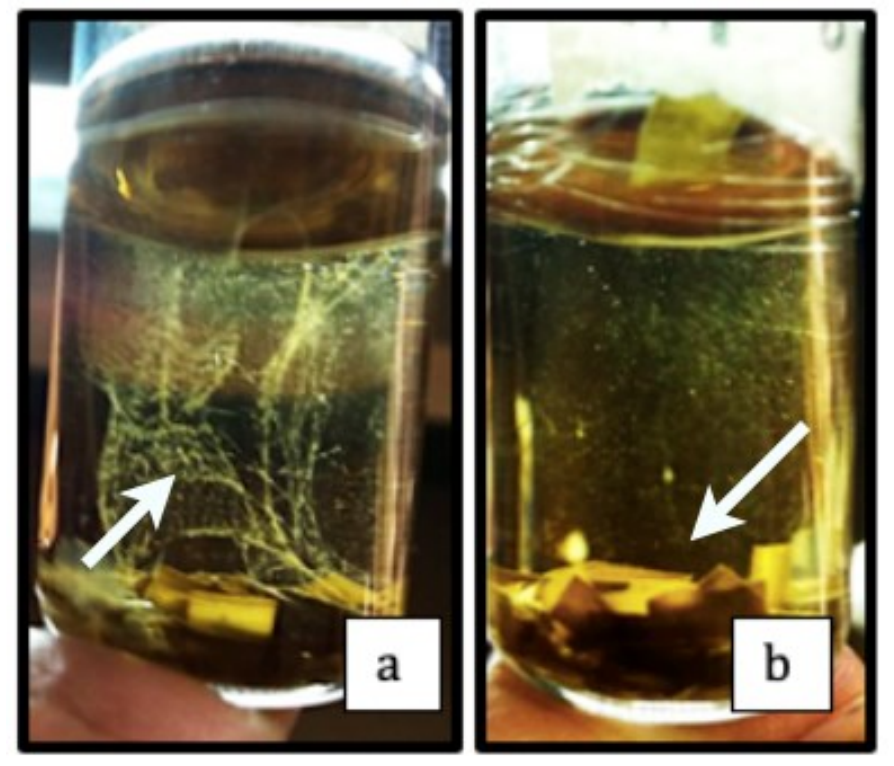

Figure 7. Formation of the ectoplasmic nets $(\mathrm{EN})$ in medium with carabao grass extract.
The EN formations were seen suspended on the leaves floating at the surface of the medium (Fig. 7A) and with those settled at the bottom of the vial (Fig. 7B).

The sterilized grass blades on a vial with $50 \%$ sea water mimicked the original environment of the organism. Isolates kept preserved in this medium were capable of lasting up to 4 months making this method useful and cost effective for the maintenance of the labyrinthulid species. Consequently, the Labyrinthula cells grew with ease when subcultured on fresh agar media taken straight from the vials.

\section{Growth in Extract-based Media}

There were two customized agar media used, MGPYA (GPYA with mangrove leaves extract), and CGPYA (GPYA with carabao grass extract). Growth in both media was analyzed and the results showed no significant differences compared to the control base medium, GPYA. Thus, it could be inferred that the labyrinthulid isolates could grow on solid media as long as the necessary nutrients were present but their growth was preferably better in liquid media such as the broth medium with carabao leaves. The agar components in the solid media could probably hinder the movement and growth of the organism.

\section{Conclusion}

Labyrinthula species are very common in the marine ecosystem, however, their mode of growth and physiology are difficult to understand along with their prolonged cell viability. They have been isolated from various habitats (marine and estuarine seawater, decaying mangrove leaves and senescent microalgae), yet the majority of these labyrinthulid species remain poorly described owing to their complicated isolation and cultivation procedures. In this study, their growth and preservation on carabao grass has been proven effective for their long-term viability up to 4 months. Agar block method was shown to be the best purification technique for their successive growth and cultivation. As these organisms are important ecologically, further scientific researches have to be undertaken for more improved purification techniques and culture maintenance of this elusive organism. 


\section{Acknowledgement}

The authors would like to express gratitude to Dr. Irineo J. Dogma, Jr. for his valuable advices, insights, and guidance in the conduct of this experiment.

\section{Literature Cited}

Bennett, R.M., D. Honda, G.W. Beakes, \& M. Thines, 2017. Labyrinthulomycetes. Handbook of the Protists, Springer International Publishing AG, p. 1-36.

Bigelow, D., M. Olsen \& R. Gilbertson, 2005. Labyrinthula terrestris sp. nov., a new pathogen of turf grass. Mycologia, 97: 185-190.

Bockelmann, A., K. Beining \& T. Reusch, 2012. Widespread occurrence of endophytic Labyrinthula spp. in northern European eelgrass Zostera marina beds. Marine Ecology Progress Series, 445: 109-116.

Chamberlain, A.H. \& S. T. Moss, 1988. The thraustochytrids: a protist group with mixed affinities. BioSystems, 21: 341349.

Garcias-Bonet, N., T. Sherman, C.M. Duarte \& N. Marba, 2011. Distribution of pathogenicity of the protist Labyrinthula sp. in western Mediterranean seagrass meadows. Estuaries and Coasts, 34: 1161-1168.

Kumon, Y., T. Yokochi \& T. Nakahara, 2005. High yield of long-chain polyunsaturated fatty acids by labyrinthulids on soybean lecithin-dispersed agar medium. Applied Microbiology and Biotechnology, 69: 253-258.

Kumon, Y., T. Yokochi, T. Nakahara, M. Yamaoka \& K. Mito, 2002. Production of long-chain polyunsaturated fatty acids by monoxenic growth or labyrinthulids on oil-dispersed agar medium. Applied Microbiology and Biotechnology, 60: 275-280.

Kumon, Y., R. Yokoyama, H. Zinia, T. Yokochi, D. Honda \& T. Nakahara, 2005. A new labyrinthulid isolate that produces only docosahexaenoic acid. Marine Biotechnology, 8: 170-177.

Leander, C.A. \& D. Porter, 2001. The labyrinthulomycota is comprised of three distinct lineages. Mycologia, 93: 459464.

Leander, C.A., D. Porter \& B.S. Leander, 2004. Comparative morphology and molecular phylogeny of aplanochytrids (Labyrinthulomycota). European Journal of Protistology, 40: 317-328.

Leaño, E.M. \& V. Damare, 2012. Chapter 12: Labyrinthulomycota. In Gareth Jones, E.B. \& Pang, K. (Eds) Marine fungi: and fungal-like organisms (pp.210243). Boston, MA: De Gruyter.
Lindholm, T., C. Lindqvist, \& C. Sjöqvist, 2016. Occurrence and activity of slime nets, Labyrinthula sp. among aquatic plants in cold and oligohaline Baltic Sea waters. Annales Botanici Fennici, 53: 139-143.

Martin, D., E. Boone, M. Caldwell, K. Major, A. Boettcher, 2009. Liquid culture and growth quantification of the seagrass pathogen, Labyrinthula sp. Mycologia, 101: 632635.

Perveen, Z., H. Ando, A. Ueno, Y. Ito, Y. Yamamoto, Y. Yamada, T. Takagi, T. Kaneko, K. Kogame \& H. Okuyama, 2006. Isolation and characterization of a novel thraustochytrid-like microorganism that efficiently produces docosahexaenoic acid. Biotechnology Letters, 28: 197-202.

Raghukumar, S., 2002. Ecology of the marine protists Labyrinthulomycetes (thraustochytrids and labyrinthulids). European Journal of Protistology, 38: 127-145.

Sakata, T., T. Fujisawa \& T. Yoshikawa, 2000. Colony formation and fatty acid composition of marine labyrinthulid isolate grown on agar media. Fisheries Science, 66: 84-90.

Sparrow, F.K., 1973. Mastigomycotina (zoosporic fungi): In Ainsworth G.C., Sparrow, F.K. and Sussman, A.S. (eds): The fungi, an advanced treatise. Vol IV B, pp. 64-73. Academic Press, New York.

Wahid, M.I., T. Yoshikawa \& T. Sakata, 2007. Bacteriolytic activity and growth of marine isolates of labyrinthulids on dead bacterial cells. Fisheries Science, 73: 1286-1294.

Wang, Q., H. Ye, Y. Xie, Y. He, B. Sen \& G. Wang, 2019. Culturable diversity and lipid production profile of Labyrinthulomycete protists isolated from coastal mangrove habitats of China. Marine Drugs, 17: 286-285.

Yadagiri, K.K., J. Kerrigan, J. \& S.B. Martin, 2012. Improved methods for axenic culture of Labyrinthula terrestris, causal agent of rapid blight of turfgrasses. Canadian Journal of Microbiology, 58: 1230-1235.

Yokochi, T., T. Nakahara, T. Higashihara, M. Yamaoka \& R. Kurane, 2001. A new isolation method for labyrinthulids using a bacterium, Psychrobacter phenylpyruvicus. Marine Biotechnology, 3: 68-73. 